Decision Making in Manufacturing and Services

VOL. $2 \bullet 2008 \bullet$ No. 1-2 • PP. 47-62

\title{
Partial Coordination May Increase Overall Costs in Supply Chains
}

\author{
Waldemar Kaczmarczyk*
}

\begin{abstract}
This paper presents a computational study to evaluate the impact of coordinating production and distribution planning in a two-level industrial supply chain. Three planning methods are compared. The first emulates the traditional way of planning. The two other coordinate plans of the supplier and of all the buyers according to the Vendor Managed Inventory (VMI) approach. The monolithic method solves a single model describing the entire optimization problem. The sequential method copies the imperfect VMI practice. All three methods are implemented by means of Mixed Integer Programming models. The results presented prove that the right choice of planning method is very important for overall cost of the supply chain. In contrast to the previous research, it turned out that information sharing without full coordination may even lead to increase in the overall cost. For some companies applying the VMI approach, developing exact models and solving them almost optimally may therefore be very important.
\end{abstract}

Keywords: supply chain, production, distribution, lot-sizing, coordination, mixed integer programming

Mathematics Subject Classification: 90B30, 90B06, 90C11

Revised: 7 November 2008

\section{INTRODUCTION}

In the traditional supply chain, the buyer plans his replenishment accordingly to the end-user demand, has to bear delivery and inventory holding costs, but ignores supplier capacity, set-up and inventory holding costs. The supplier has no information about the end-user demand and plans his production according to buyer orders. There is little place for coordination in this approach.

New cooperation relationships between buyers and suppliers that allow better coordination have gained in popularity over recent years. They share some common characteristics (Lee and Chu, 2005):

1. The buyer shares end-user demand information with the supplier,

2. The supplier has full inventory control in the supply chain, keeps the inventory and bears the risk of demand uncertainty.

\footnotetext{
* Department of Operations Research and Information Technology, AGH University of Science and Technology, Krakow, Poland, E-mail: waldek@agh.edu.pl
} 
One of them is called Vendor Managed Inventory (VMI). Under the VMI approach, downstream companies, buyers, share end-user demand information with the upstream companies, suppliers, who should then make stock level decisions for both the upstream and the downstream. To discourage suppliers from moving to the buyers as much inventory as is allowed, VMI contracts sometimes permit buyers to return excess goods to the supplier or make supplier responsible for inventory holding costs at buyers' site. VMI is also sometimes referred to as the Continuous Replenishment Program (CRP) or the Continuous Product Replenishment program (CPR) in the packaged-goods industry (Lee and Chu, 2005).

Under the terms of a VMI contract, the supplier has the opportunity to coordinate his production with distribution. Unfortunately, both of these planning problems belong to a family of complex lot-sizing problems. The integrated production and distribution problem may not be easier. There are several surveys on coordination of production and distribution in supply chains, e.g. Thomas and Griffn (1996), Sarmiento and Nagi (1999), Erengüç et al. (1999).

There are also some computational studies evaluating impact of coordination. Sahin and Robinson Jr. (2005) evaluated different strategies in the make-to-order environment: four with none or partial information sharing, with none or partial coordination, and one with full information sharing and full coordination. For various data parameters, i.e. the number of items, supplier equipment changeover cost, delivery fixed cost or demand variability, they reported an average of $47.58 \%$ system wide cost reduction when moving from a traditional strategy to a fully coordinated supply chain. In their opinion, tighter coordination among channel members may provide a more effective lever for cost improvement than information sharing.

Chandra and Fisher (1994) report from 3\% to $20 \%$ reduction in the total operating cost with coordination for various data sets. Furthermore, they concluded that the value of coordination increases when the supplier production capacity decreases on the one hand, or the length of the planning horizon, the number of products or customers increase on the other hand.

In this paper, three planning methods are compared. The first emulates the traditional way of planning, where all the buyers make their replenishment decisions and the supplier plans his production to satisfy buyers' demand. The two other coordinate plans of the supplier and of all the buyers according to the Vendor Managed Inventory (VMI) approach. The monolithic method solves a single model describing the entire optimization problem. The sequential method copies the imperfect VMI practice, and first schedules the suppliers' production for the aggregated demand of all the buyers and then solves the distribution problem. Both methods assume that a single decision maker has full information and all decisions in his hand, different is only the way he/she makes use of it. The monolithic method tries to solve one big model, which is difficult due to its complexity. The sequential method decomposes the problem into two subproblems, similarly to the traditional method, but solves them in a different order, more natural for the supplier.

The traditional method does not coordinate production and distribution in any way. The monolithic method strives for full coordination. The sequential method applies partial coordination, i.e. plans production simultaneously for all the buyers but 
ignores dependence of the production schedule on distribution costs and constraints. The objective of this study is to investigate the impact of these planning methods on the overall supply chain cost at various parameters settings.

The model examined in this paper was inspired by Miodońska (2006) who discussed distribution planning in the automotive industry. Automobile manufacturers, buyers of car components, usually work in the just-in-time fashion, i.e. do not do any batching. This leads to rather stationary product mix and stationary demand for components. Therefore, component suppliers cooperating with car manufacturers in the VMI fashion do not have to face lumpy demand. On the other hand, most suppliers have to deal with relatively high set-up costs and fixed delivery costs, which forces batching of production and deliveries.

In the next section, the monolithic Mixed Integer Programming (MIP) model of the problem at hand is presented. Section 3 describes decomposition procedures, the traditional and sequential methods. Section 4 presents data parameters and section 5 provides results of experiments. Conclusions are made in section 6 .

\section{MONOLITHIC METHOD}

In the problem at hand, several components are produced over time by a single supplier. Setup costs are reasonable with one set-up a day at the most. Joint deliveries of all components to several buyers are done with tracks of various capacity, fixed cost and delivery time. The daily demand for each component of each buyer is known. The problem is to schedule production and distribution so as to minimize the total cost of the inventory, production and transportation set-ups. Table 1 defines all the parameters and Table 2 provides all the variables in the model.

Table 1. Data parameters

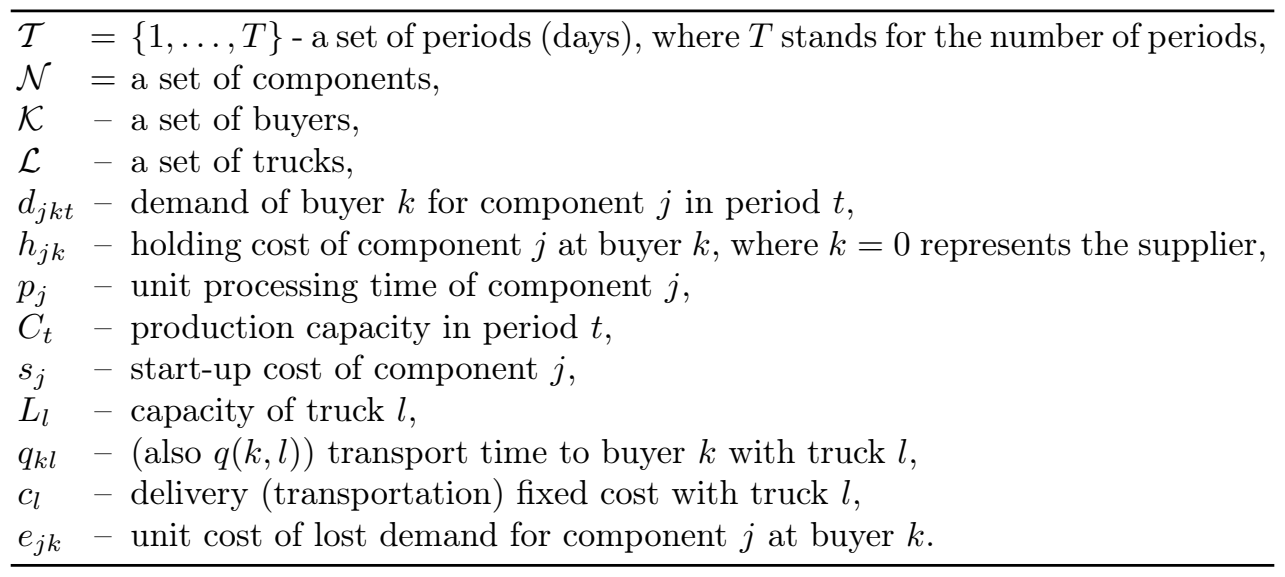


Safety stocks required by buyers and initial inventories can be subtracted from demand of initial periods and therefore do not have to be taken into consideration. All components have the same size and weight.

Table 2. Variables

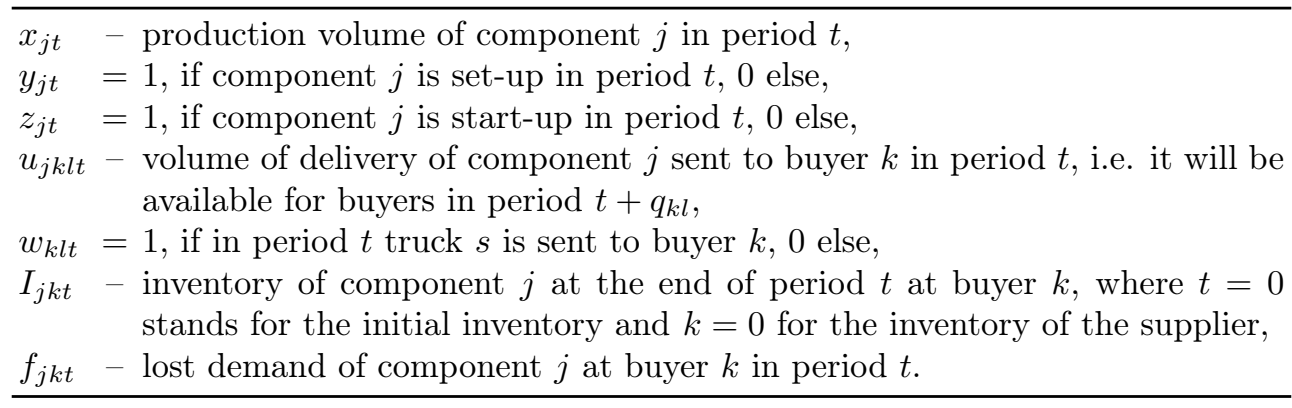

The set-up variables $y_{j t}$ describe the state of the machines, i.e. which components can be produced on a given day. The start-up variables $z_{j t}$ describe changes in the state of the machines, i.e. if $y_{j, t-1}=0$ and $y_{j t}=1$ then $z_{j t}=1$, otherwise $z_{j t}=0$.

The following monolithic model (1) describes all the cost factors and all the constraints of the integrated production-distribution problem.

$$
\begin{aligned}
& \min \sum_{j \in \mathcal{N}} \sum_{t \in \mathcal{T}}\left(s_{j} z_{j t}+h_{j 0} I_{j 0 t}+\sum_{k \in \mathcal{K}}\left(h_{j k} I_{j k t}+e_{j k} f_{j k t}\right)\right) \\
& +\sum_{k \in \mathcal{K}} \sum_{l \in \mathcal{L}} \sum_{t \in \mathcal{T}}\left(c_{l} w_{k l t}+\sum_{j \in \mathcal{N}} q_{k l} h_{j k} u_{j k l t}\right) \\
& \text { s.t. } \quad I_{j k, t-1}+\sum_{l \in \mathcal{L}} u_{j k l, t-q(k, l)}-d_{j k t}+f_{j k t}=I_{j k t}, \quad t \in \mathcal{T}, j \in \mathcal{N}, k \in \mathcal{K} \text {, } \\
& \sum_{j \in \mathcal{N}} u_{j k l t} \leq L_{s} w_{k l t}, t \in \mathcal{T}, k \in \mathcal{K}, l \in \mathcal{L}, \\
& I_{j 0, t-1}+x_{j t}-\sum_{k \in \mathcal{K}} \sum_{l \in \mathcal{L}} u_{j k l t}=I_{j 0 t}, \quad t \in \mathcal{T}, j \in \mathcal{N}, \\
& x_{j t} \leq B_{j t} y_{j t}, \quad t \in \mathcal{T}, j \in \mathcal{N}, \\
& \sum_{j \in \mathcal{N}} y_{j t} \leq 1, \quad t \in \mathcal{T}, \\
& y_{j t}-y_{j, t-1} \leq z_{j t}, \quad t \in \mathcal{T}, j \in \mathcal{N}, \\
& x_{j t}, u_{j k l t}, I_{j k t} \geq 0, \quad t \in \mathcal{T}, j \in \mathcal{N}, \\
& k \in \mathcal{K}, l \in \mathcal{L}, \\
& y_{j t}, z_{j t}, w_{k l t} \in\{0,1\}, \quad t \in \mathcal{T}, j \in \mathcal{N}, \\
& k \in \mathcal{K}, l \in \mathcal{L} .
\end{aligned}
$$


$B_{j t}=\min \left\{C_{t} / p_{j t}, \sum_{l=t, \ldots, T} d_{j l}\right\}$ is a number not smaller than $x_{j t}$. The objective function (1a) summarizes all the costs: the costs of the supplier, then of all the buyers, and finally the costs of distribution. Included are the costs of the "inventory on wheels", i.e. during transport, to prevent any unreasonable preference for slower cars. Constraint (1b) preserves balance of deliveries, inventory and end-user demand at buyers' sites. Equation (1c) requires delivery set-up if delivery volume of any component is positive, and preserves that capacity of a selected truck is not exceeded. Constraint (1d) preserves balance of production, inventory and deliveries at supplier site. Constraint (1e) requires start-ups for component in periods in which production of that component occurs. Equation $(1 \mathrm{~g})$ couples set-up and start-up variables. Equations describing the supplier's production process (1d)-(1g) are typical for the Continuous Setup Lot-Sizing Problem (Drexl and Kimms, 1997).

\section{DECOMPOSITION METHODS}

The monolithic model presented in the previous section simultaneously determines production and distribution plans. In practice, it is very difficult to solve such a big model. Therefore, most companies decompose the entire problem into subproblems which are assumed to be easier to solve and to guarantee acceptable solutions. This paper discusses two methods of vertical decomposition, i.e. hierarchical approaches, which solve subproblems consecutively and a solution of each subproblem limits the decision space of the subproblems solved later. There are three important elements of vertical decomposition: separation of decisions, sequence of subproblems and type of instruction limiting further solutions, i.e. decisions passed from subproblems solved earlier to subproblems solved later. Separation of decisions in the problem at hand is obvious. There are two subproblems reflecting the natural business process:

1. Production planning, i.e. the decisions when to start-up production of every component and how much to produce,

2. Distribution planning, i.e. the decisions when to send a truck of which capacity to a buyer and how many components to deliver.

There are two possible sequences of these subproblems. In the traditional method, first the buyers solve the distribution problem. The sequential method, assuming the VMI approach, copies the industrial practice and begins with determining of the production plan. This is a more natural way of planning for the supplier, as production actually precedes distribution and most suppliers already have advanced production planning procedures.

The following sections present MIP models describing the decision process in the sequential method and indicating differences against the traditional method.

\subsection{PRODUCTION PLANNING}

Model (2) describes the objective function and all constraints of the production planning model applied to schedule suppliers' production in the sequential method. The VMI approach enables collecting demand forecasts from all the buyers and planning 
production of components according to end-user demand and not according to buyers' orders. Thus the supplier can coordinate production of components for all the buyers and can avoid lumpy demand generated by buyers' orders.

$$
\begin{array}{rlrl}
\min \sum_{j \in \mathcal{N}} \sum_{t \in \mathcal{T}}\left(s_{j} z_{j t}+h_{j 0} I_{j 0 t}+e_{j} f_{j t}\right) & & \\
\text { s.t. } I_{j 0, t-1}+x_{j t}-\sum_{k \in \mathcal{K}} d_{j k, t+Q}+f_{j t} & =I_{j 0 t}, & & t \in \mathcal{T}, j \in \mathcal{N}, \\
x_{j t} \leq B_{j t} y_{j t}, & & t \in \mathcal{T}, j \in \mathcal{N}, \\
\sum_{j \in \mathcal{N}} y_{j t} \leq 1, & & t \in \mathcal{T}, \\
y_{j t}-y_{j, t-1} \leq z_{j t}, & t \in \mathcal{T}, j \in \mathcal{N}, \\
x_{j t}, I_{j t} \geq 0, & t \in \mathcal{T}, j \in \mathcal{N}, \\
y_{j t}, z_{j t} \in\{0,1\}, & t \in \mathcal{T}, j \in \mathcal{N},
\end{array}
$$

In this model, only balance constraints $(2 \mathrm{~b})$ have been modified in comparison with the monolithic model. Delivery volumes had to be replaced by the aggregated demand of all the buyers. To make such aggregation possible, assumptions have to be made about delivery time. This paper assumes that the whole demand is shifted by the maximum delivery time $Q=\max _{k \in \mathcal{K}, l \in \mathcal{L}} q_{k l}$. Furthermore, variable $f_{j t}$ represents the total lost demand of all the buyers.

\subsection{DISTRIBUTION PLANNING}

When the production plan is fixed, production volumes can be passed as instruction to distribution planning described in the model (3). The inventory and lost demand values from production planning can be ignored as distribution planning can more accurately calculate values of these variables employing exact delivery volumes.

$$
\begin{array}{rlrl}
\min \sum_{t \in \mathcal{T}} \sum_{k \in \mathcal{K}}\left(\sum_{j \in \mathcal{N}}\left(h_{j k} I_{j k t}+e_{j k} f_{j k t}\right)+\sum_{l \in \mathcal{L}}\left(c_{l} w_{k l t}+\sum_{j \in \mathcal{N}} q_{k l} h_{j k} u_{j k l t}\right)\right) & \\
\text { s.t. } I_{j k, t-1}+\sum_{l \in \mathcal{L}} u_{j k l, t-q(k, l)}-d_{j k t}+f_{j k t}=I_{j k t}, & t \in \mathcal{T}, j \in \mathcal{N}, k \in \mathcal{K}, \\
u_{j k l t} \leq L_{s} w_{k l t}, & t \in \mathcal{T}, k \in \mathcal{K}, l \in \mathcal{L}, \\
I_{j 0, t-1}+x_{j t}^{*}-\sum_{k \in \mathcal{K}} \sum_{l \in \mathcal{L}} u_{j k l t}=I_{j 0 t}, & t \in \mathcal{T}, j \in \mathcal{N}, \\
x_{j t}, u_{j k l t}, I_{j k t} \geq 0, & & t \in \mathcal{T}, j \in \mathcal{N}, \\
w_{k l t} \in\{0,1\}, & & t \in \mathcal{K}, k \in \mathcal{T}, k \in \mathcal{K}, l \in \mathcal{L},
\end{array}
$$


The only difference between the constraints in this model and in the monolithic model is in parameter $x_{j t}^{*}$ which represents production volumes calculated in the production model and is not subject to changes in the distribution model.

The above distribution planning problem cannot be solved separately for all the buyers as they all depend on common production volumes, i.e. they share suppliers component inventories. After simultaneous planning of production for all the buyers this may be seen as another form of coordination. However, the production and distribution schedules continue to remain uncoordinated. Therefore, the sequential method represents only a partial coordination approach.

\subsection{TRADITIONAL METHOD}

Traditional planning follows order reversed against the sequential method. First, all the buyers completely independently make joint replenishment decisions and send orders to the supplier. The buyers take into account no suppliers' capacity utilization, start-up costs of machines or orders from the other buyers.

Supplier has to adjust his production plan to the buyers' orders. The only way that the buyers can make this task for the supplier a little easier is to share with him the information about planned replenishment orders as early as possible. This paper assumes that the supplier knows orders for the same horizon as end-user demand forecasts used in the monolithic and sequential methods.

\section{DATA PARAMETERS}

The objective of this study is to investigate the impact of the three planning methods on the overall supply chain cost at various parameters settings.

The data for two demand scenarios and truck characteristics have been collected by Miodońska (2006) for the automotive industry. In all the scenarios, there are two components, one supplier and three buyers, i.e. car manufacturers, practically in the same distance to the supplier. The planning horizon is 30 days long, but effective planning horizon is 2-3 days shorter due to long delivery times.

Truck characteristics are given in table 3 Note high variability of the fixed cost-capacity ratio.

Table 3. Characteristics of trucks (Miodonska 2006)

\begin{tabular}{ccc}
\hline Truck & $\begin{array}{c}\text { Capacity-fixed cost ratio } \\
\left(L_{l} / c_{l} * 100 \%\right)\end{array}$ & $\begin{array}{c}\text { Delivery time } \\
\text { [days] }\end{array}$ \\
\hline 1 & $41 \%$ & 2 \\
2 & $100 \%$ & 2 \\
3 & $234 \%$ & 3 \\
4 & $290 \%$ & 3 \\
\hline
\end{tabular}

The second truck is the most frequently used and serves as a reference 
In the study, 144 test problems are formed from two demand scenarios and all combinations of the following parameters: lost demand unit cost $e_{j} \in\{40,400\}$, start-up costs $s_{j} \in\{50,100,200\}$, fixed delivery cost of the second truck $c_{2} \in\{125,250,500\}$ (the most widely used), suppliers capacity utilization $u \in\{60 \%, 70 \%, 80 \%, 90 \%\}$.

Utilization $u$ is defined as the total demand divided by capacity of 27 days available for production. Production on day 28 does not make any sense as the fastest truck needs 2 days to deliver the components to any buyer.

The values of lost demand unit cost $e_{j} \in\{40,400\}$ require some explanations. Higher value should actually prevent any lost demand in the solutions as its is higher than the set-up cost for both production and delivery (with the smallest truck). Thus, high values do not have any economical justification. Smaller value will allow more accurate comparison of the results in a case when lost demand is inevitable.

In the practice of the automotive industry, both settings might be accurate. When the supplier plans his operations for the already accepted end-user demand volumes, the indispensable inventory levels at the buyers' sites must be guaranteed. On the other hand, the buyers, i.e. car manufacturers, sometimes face unexpected short-horizon demand fluctuations and ask suppliers to make deliveries earlier or to deliver more than previously agreed. Under such circumstances, the supplier has no obligation to deliver the ordered quantities but attempts to satisfy the buyers' unplanned demand. That situation will be modelled by a smaller value of lost demand unit cost.

The unit processing time $p_{j}$ and the unit holding costs $h_{j k}$ have been set to 1 for all the components and all the buyers. Therefore, the values of lost demand unit cost $e_{j}$, start-up costs $s_{j}$ and fixed delivery cost $c_{l}$ express also the ratio to unit holding cost.

\section{EXPERIMENTAL RESULTS}

All the three methods analyzed in this paper have been implemented in ILOG OPL Development Studio 6.1 using ILOG CPLEX 11 to solve MIP problems (Fig. 1). All the test problems have been solved on a computer with the Intel T1300 $1.66 \mathrm{MHz}$ processor with 10 minutes time limit. In the decomposition methods production planning was solved optimally in less than a minute and the rest of time was spent on solving the distribution (replenishment) problem.

The percentage duality gap between the best integer solution $f^{*}$ and the linear relaxation $f^{R}$ was calculated as $\left(f_{*}-f^{R}\right) / f^{R}$. For the methods consisting of several models, total $f^{*}$ and $f^{R}$ were calculated as a sum of $f^{*}$ and $f^{R}$ of all these models. The average values of duality gap are given in Figure 1. For the monolithic method they ranged from $20.2 \%$ to $36.5 \%$, for the sequential method from $5.4 \%$ to $17.8 \%$, and for the traditional method from $0.8 \%$ to $8.6 \%$.

The most dramatic effect visible in this figure is the high average duality gap of the monolithic method, equal to $29.1 \%$. It is three times higher than for the sequential method $(10.7 \%)$ and six times higher than for the traditional method $(4.9 \%)$.

Furthermore, if fixed delivery costs are high, the distribution problem becomes more important and the traditional method is completed with a very small gap. 
If fixed delivery costs are small, the sequential method has duality gap almost as small as the traditional one. The suppliers' start-up costs do not seem to have any significant impact on the complexity of the problem. These results are not surprising. Complexity of the monolithic model is much higher and in case of high delivery costs the traditional method, which initially tries to minimize these costs, should achieve better results.

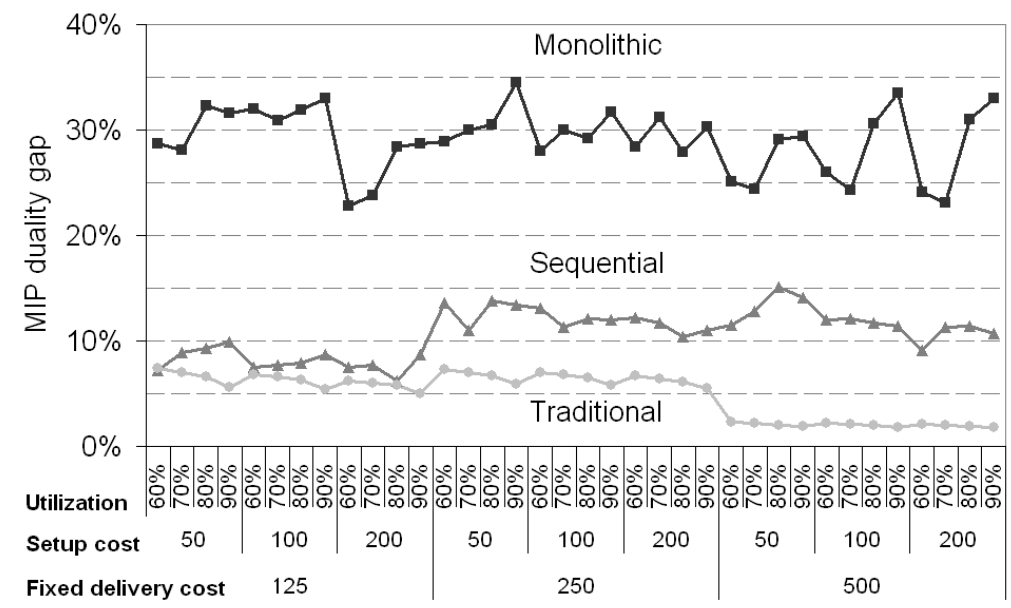

Fig. 1. Average MIP duality gap at small lost demand unit costs

Figures 2 and 3 give values of all the cost factors of the entire supply chain. The first figure gives average values for the test problems with small lost demand unit cost as percentage ratio to the overall cost determined with the traditional method.

The most significant finding in Figure 2 is the average $17 \%$ decrease of overall cost possible with the monolithic method. The sequential method is also better than the traditional one (in average by $6 \%$ ).

Both decomposition methods guarantee small values of costs minimized in the first step of planning. The sequential method generated small start-up and inventory costs, the traditional one produced small delivery costs.

The monolithic method surpasses the two other methods primarily in minimizing lost demand. It is a very good news for the supplier, as satisfaction of the buyer is at least as important as the cost effect (assuming that the supplier is able to build and solve the monolithic model). 


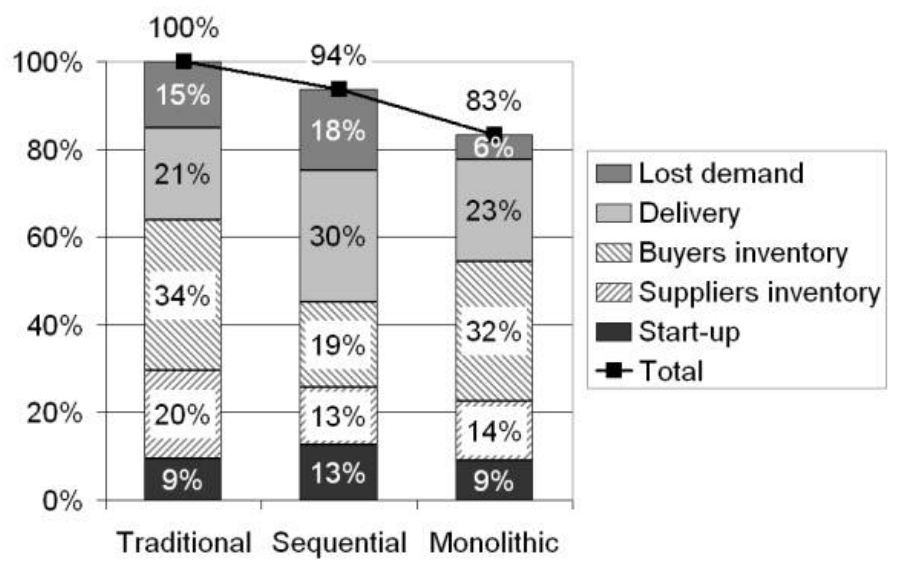

Fig. 2. Total cost factors at small lost demand unit costs, as ratio to total cost obtained with the traditional method

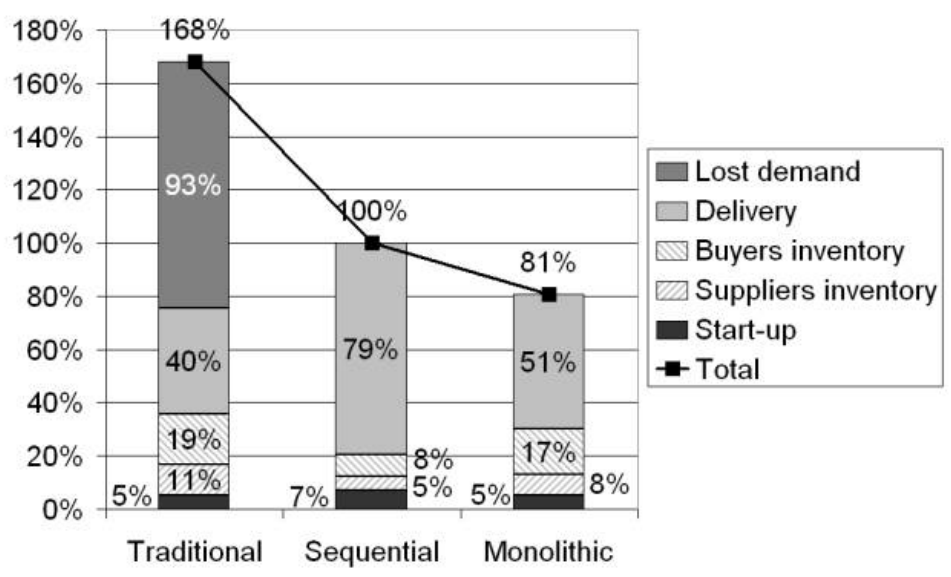

Fig. 3. Total cost factors at high lost demand unit costs, as ratio to total cost obtained with the sequential method

Figure 3 presents cost values for the test problems with prohibitive lost demand costs expressed as percentage ratio to the overall cost determined with the sequential method. First of all, the traditional method fails to eliminate lost demand. This cannot be surprising as the traditional method completely ignores supplier capacity limits while planning component deliveries. Furthers details are given in Figure 4. 
Both methods implementing the VMI approach eliminate lost demand completely. It is interesting to compare their results without lost demand: delivery costs dominate all other cost factors then. Obviously, successful coordination requires much more frequent deliveries with smaller trucks. However, the monolithic method results in twice as high inventories and a little lower start-up costs. Finally, the monolithic method guarantees on average $19 \%$ lower overall cost than of the sequential one.

Figure 4 presents when exactly the traditional method fails to eliminate lost demand. It seems that getting low lost demand with this method is only possible at small fixed delivery costs and low utilization. High costs and high utilization may lead even to $10 \%$ lost demand.

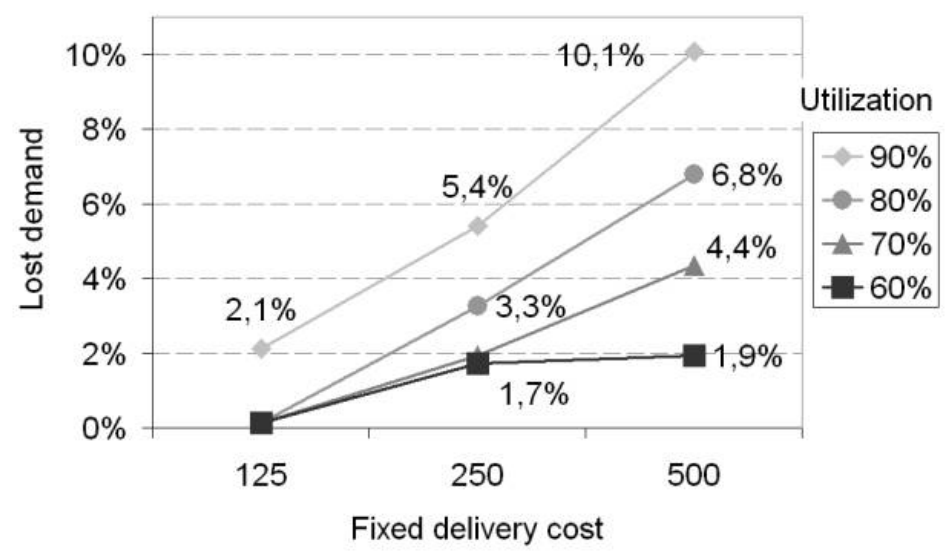

Fig. 4. Impact of suppliers' capacity utilization and delivery fixed cost on percentage of lost demand at high lost demand unit costs

Figures 5 and 6 present the relationship between the supply chain overall cost and delivery fixed cost. Both charts present ratio of the overall supply chain cost obtained with the given method to the overall cost obtained with the traditional method. Figure 5 gives the results at small lost demand unit cost. It seems that fixed delivery costs do not have significant impact except for the sequential method. This may be easily explained as this method ignores delivery cost in the first planning step. When lost demand unit costs are high (see Fig. 6), the situation is completely different. Now almost any change in unit costs significantly changes the total cost.

Figures 7 and 8 give the relationship between the total supply chain cost and the supplier' capacity utilization. The values of costs for all the methods are given as the ratio to the costs obtained with the traditional method.

Figure 7 gives the results at small lost demand unit costs. This is probably the most interesting chart in this paper. It shows that for small utilization of suppliers' capacity (about 60\%), the sequential method applying VMI approach gives $9 \%$ worse results than the traditional method. 


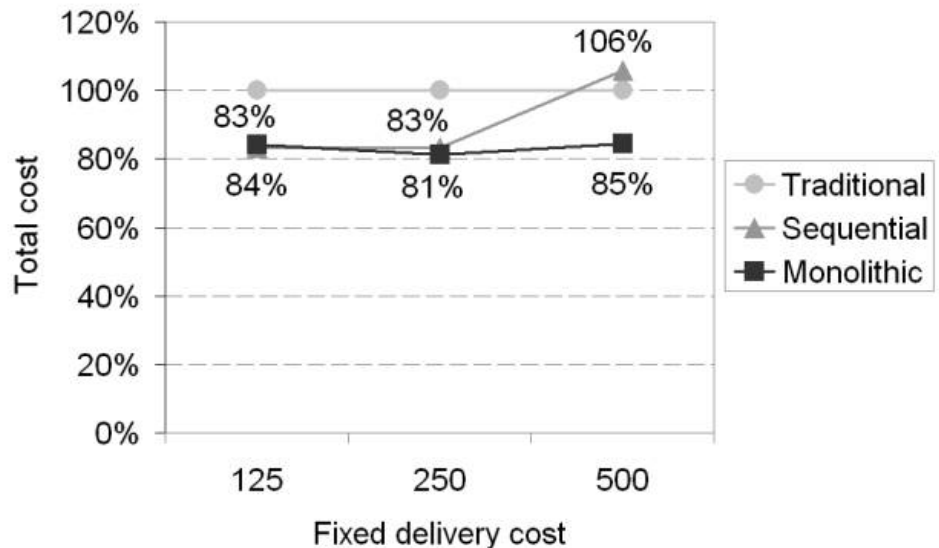

Fig. 5. Impact of delivery fixed cost on the total supply chain cost at small lost demand unit costs, as the ratio to the total cost obtained with the traditional method

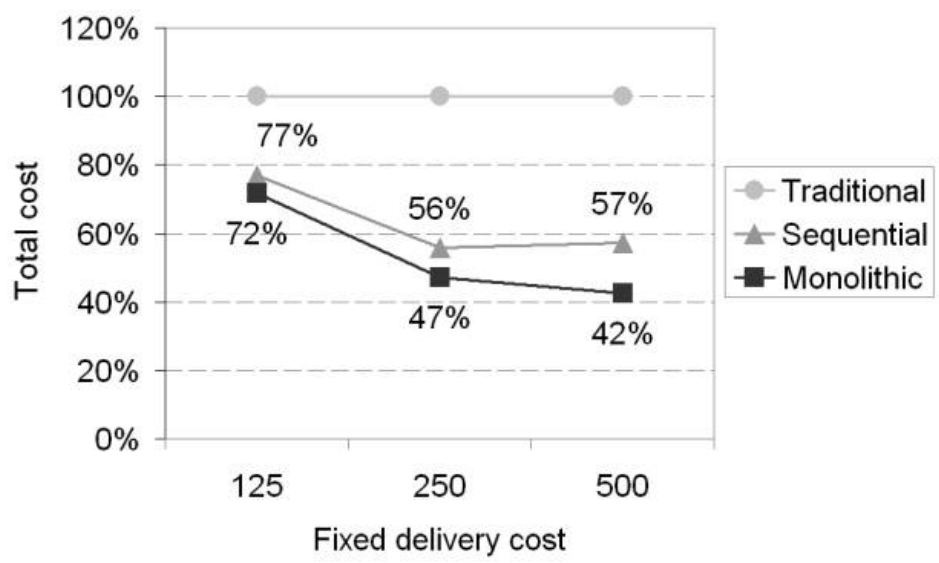

Fig. 6. Impact of delivery fixed cost on the total supply chain cost at high lost demand unit costs, as the ratio to the total cost obtained with the traditional method 


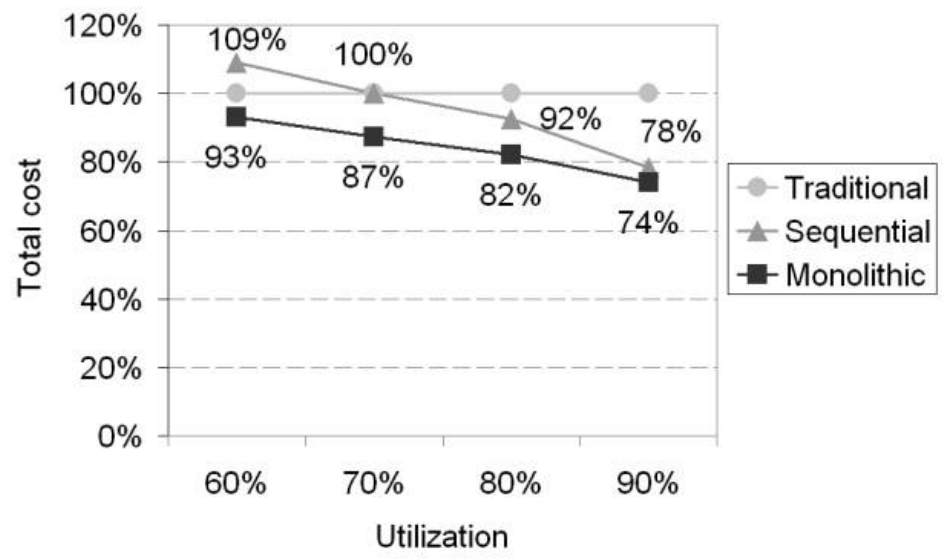

Fig. 7. Impact of suppliers' capacity utilization on the total supply chain cost at small lost demand unit costs, as the ratio to the total cost obtained with the traditional method

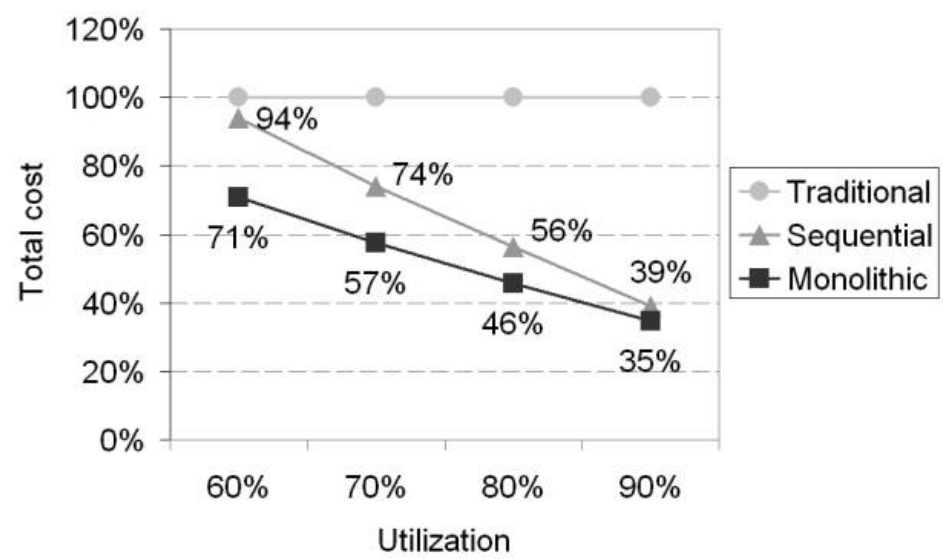

Fig. 8. Impact of suppliers' capacity utilization on the total supply chain cost at high lost demand unit costs, as the ratio to the total cost obtained with the traditional method 
For the suppliers of car components, it is a very bad news. On one hand, they have to accept low utilization of their resources to guarantee high customer service. On the other hand, they are forced to cooperate in VMI fashion. But under such circumstances, they obviously risk increase in their operational costs. This is exactly the opposite of what should be expected. Coordination enabled by VMI contracts intends to decrease operational costs and not to increase them.

The good news is that for low utilization the traditional method is not much worse than the monolithic one, with only $7 \%$ difference. Similarly, for high utilization, the sequential method seems to be almost as good as the monolithic one. The difference is only $4 \%$ and both VMI methods yield much better results than the traditional one. The sequential method is $22 \%$ better and the monolithic one as much as $26 \%$.

The general tendencies are easy to explain. The traditional method starts with minimizing distribution costs which are dominant in case of low utilization of suppliers' resources. In the opposite case, the production schedule is more important and that is why the sequential method minimizing first that part of the problem is better for high utilization.

If lost demand unit cost is high (see Fig. 8), the chart looks very similar with the difference that all values of the VMI methods are always better than those of the traditional method. For small utilization, the improvement is only $6 \%$.

The relationship between lost demand and fixed delivery cost at small lost demand unit costs is given in Figure 9. As expected, lost demand increases when delivery cost increases. A little surprising is the high value for the sequential method and high delivery costs.

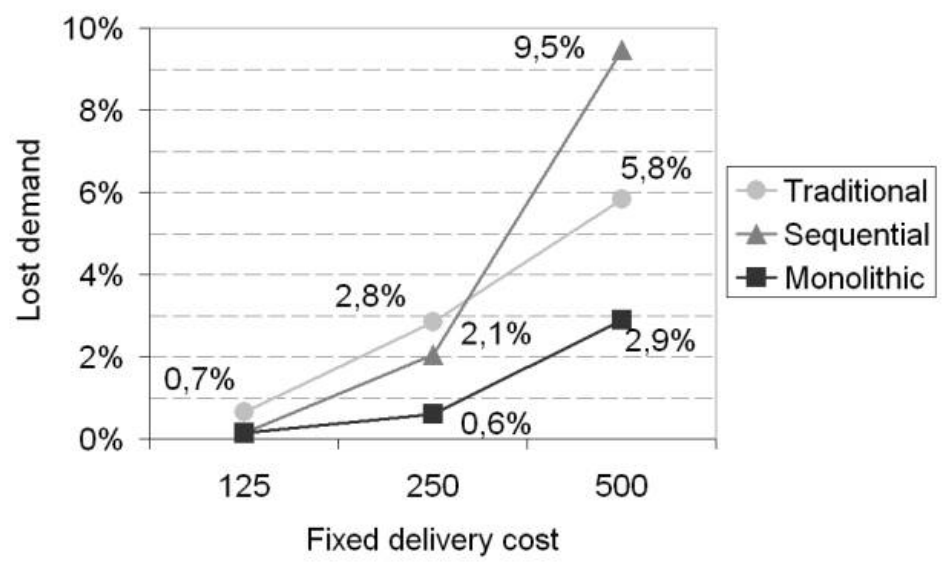

Fig. 9. Impact of delivery fixed cost on percentage of lost demand at small lost demand unit costs 
The relationship between lost demand and suppliers' capacity utilization is given in Figure 10. As expected, increasing suppliers' utilization increases volume of lost demand, more for the traditional method than for the monolithic one. The results for the sequential method are counterintuitive, but this negative relationship is very week and almost negligible.

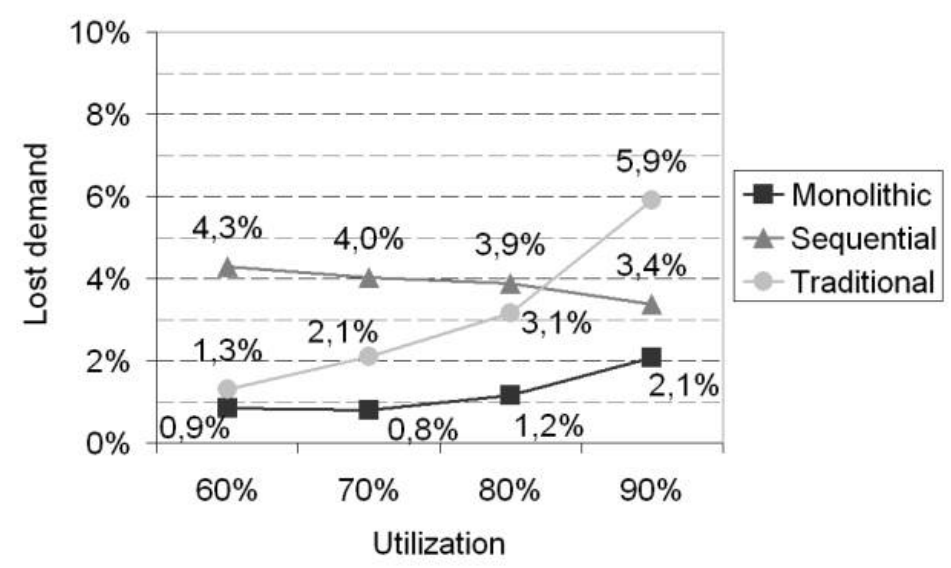

Fig. 10. Impact of suppliers capacity utilization on percentage of lost demand at small lost demand unit costs

\section{CONCLUSION}

The paper analyses operational coordination of production and distribution within an industrial supply chain. Using the Mixed Integer Programming models, three different planning methods have been studied, one emulating the traditional way of planning and two applying the Vendor Managed Inventory (VMI) approach. The former solved monolithic model of the integrated problem, the latter copied the procedures applied in the imperfect VMI practice. All the methods have been tested on several data sets for a variety of parameters .

The general conclusion is that selection of the planning method is very important and depends on actual parameter settings. For low utilization of suppliers' resources, the traditional method is not much worse than the monolithic one. For high utilization, the sequential method achieves very good results. As expected, the monolithic method is best in all cases. It is however much more complex what makes practical applications very hard.

Another conclusion is that for low utilization the sequential method may lead to increase in the overall cost in comparison with the traditional method. This is very important, because suppliers of components have often to accept low utilization of their resources to guarantee high customer service. They are also forced by the buyers to cooperate in the VMI fashion. Under such circumstances, the suppliers have to make an effort to develop full coordination procedures similarly to the monolithic method. 


\section{REFERENCES}

Chandra P. and Fisher M. L., 1994: Coordination of Production and Distribution Planning, European Journal of Operational Research 72(3): 503-517.

Drexl A. and Kimms, A., 1997: Lot Sizing and Scheduling - Survey and Extensions, European Journal of Operational Research 99(2): 221-235.

Erengüç Ş. Selçuk., Simpson N. C. and Vakharia A. J., 1999: Integrated Production/Distribution Planning in Supply Chains, European Journal of Operational Research 115: $219-236$.

Lee C. C. and Chu W. H. J., 2005: Who Should Control Inventory in a Supply Chain?, European Journal of Operational Research 164: 158-172.

Miodońska B., 2006: Koordynacja w tańcuchach dostaw (Coordination in Supply Chains), Master's thesis, AGH University of Science and Technology, Krakow, Poland, (in Polish).

Sahin F. and Robinson Jr. E. P., 2005: Information Sharing and Coordination in Make-to-order Supply Chains, Journal of Operations Management 23: 579-598.

Sarmiento A. M. and Nagi R., 1999: A Review of Integrated Analysis of Production Distribution Systems, IIE Transactions 31: 1061-1074.

Thomas D. J. and Griffn P. M., 1996: Coordinated Supply Chain Management, European Journal of Operational Research 94: 1-15. 